\title{
On Relatively Prime Odd Amicable Numbers
}

\author{
By Peter Hagis, Jr.
}

\begin{abstract}
Whether or not a relatively prime pair of amicable numbers exists is still an open question. In this paper some necessary conditions for $m$ and $n$ to be a pair of odd relatively prime amicable numbers are proved. In particular, lower bounds for $m, n, m n$ and the number of prime divisors of $m n$ are established. The arguments are based on an extensive case study carried out on the CDC 6400 at the Temple University Computing Center.
\end{abstract}

1. Introduction. Two positive integers $m$ and $n$ are said to be amicable if

$$
\sigma(m)=m+n=\sigma(n)
$$

where $\sigma(k)$ is the sum of the positive divisors of $k$. To date almost 900 pairs of amicable numbers have been found (see [1], [2], [3], [4], [7] and [8]), none of which is relatively prime. In [6] Kanold has shown that if $m$ and $n$ are relatively prime amicable numbers then $m n>4 \cdot 10^{46}$. In [5] the present author has shown that if $m$ and $n$ are relatively prime amicable numbers of opposite parity then $m n>10^{74}$. The purpose of the present paper is to improve Kanold's lower bound for $m n$ in case $m$ and $n$ are relatively prime odd amicable numbers. To be precise, we shall prove the following

THEOREM. If

$$
m=\prod_{i=1}^{M} r_{i}{ }^{b i}, \quad n=\prod_{j=1}^{N} s_{j}{ }^{c} \quad(\text { where } M+N=T)
$$

are amicable numbers such that the odd primes $r_{i}$ and $s_{j}$ are distinct then

(a) if $(m n, 15)=1$, then $T \geqq 606$ and $m n>10^{1911}$;

(b) if $(m n, 15)=5$, then $T \geqq 140$ and $m n>4 \cdot 10^{354}$;

(c) if $(m n, 15)=3$, then $T \geqq 53$ and $m n>3 \cdot 10^{118}$;

(d) if $(m n, 15)=15$, then $T \geqq 21$ and $m n>10^{67}$.

The proof involves an exhaustive, and rather exhausting, "case" study. This was carried out with the aid of the CDC 6400 at the Temple University Computing Center.

2. Some Preliminary Results. In what follows $p$ and $q$ denote primes and the notation $p^{a}|| k$ means that $p^{a} \mid k$ but $p^{a+1} \nmid k . m$ and $n$ will always be understood to be a pair of relatively prime odd amicable numbers. From (1) and (2) and the multiplicative property of $\sigma(k)$ we see that

$$
m+n=\prod \sigma\left(r^{b}\right)=\prod \sigma\left(s^{c}\right)
$$

where, for convenience, we have omitted the subscripts.

Lemma 1. If $q \mid m n$ and $p^{a} \| m n$, then $q \nmid \sigma\left(p^{a}\right)$.

Proof. If $q \mid m n$ and $q \mid \sigma\left(p^{a}\right)$, then from (3) we see immediately that $q \mid m$ and $q \mid n$. This is impossible since $(m, n)=1$.

Received August 6, 1968, revised October 10, 1968. 
Lemma 2. If $q \mid m n, p^{a} \| m n$, and $p=q k-1$ then $2 \mid a$.

Proof. If $p=q k-1$ and $a$ is odd, then $\sigma\left(p^{a}\right) \equiv 1+p+p^{2}+\cdots+p^{a} \equiv$ $1-1+1-\cdots-1 \equiv 0(\bmod q)$. Since this contradicts Lemma 1 we conclude that $a$ is even.

Now, since $m \neq n$ we have $(m+n)^{2} / m n>4$. Therefore, letting $\prod r^{b} \cdot \prod s^{c}=$ $p_{1}{ }^{a_{1}} \cdot p_{2}{ }^{a_{2}} \cdots p_{T}{ }^{a_{T}}$ where $p_{i}<p_{j}$ if $i<j$, we have from (3)

Lemma 3. If $m n=\prod_{i=1}^{T} p_{i}{ }^{a}$, then $4<\prod_{i=1}^{T} \sigma\left(p_{i}{ }^{a}\right) / p_{i}{ }^{a}$.

3. The case $(m n, 15)=1$. In the sequel we shall denote the $j$ th odd prime by $P_{j}$. If $(m n, 15)=1$ then $p_{1} \geqq 7=P_{3}$. Since $\sigma\left(p^{a}\right) / p^{a}=\left(p-p^{-a}\right) /(p-1)<$ $p /(p-1)$ we see from Lemma 3 that

$$
4<\prod_{i=1}^{T} p_{i} /\left(p_{i}-1\right) \leqq \prod_{j=3}^{T+2} P_{j} /\left(P_{j}-1\right) .
$$

It follows that if $N$ is the smallest integer such that $\prod_{j=3}^{x} P_{j} /\left(I_{j}-1\right)>4$, then $T \geqq N-2$ and $m n \geqq \prod_{j=3}^{N} P_{j}$. Making use of the CDC 6400 it was found that $N=608$ and $m n \geqq 7 \cdot 11 \cdot 13 \cdots 4483>10^{1911}$.

4. The case $(m n, 15)=5$. In this case $p_{1}=5=P_{2}$. Therefore, if $N$ is the smallest integer such that $\prod_{j=2}^{v} P_{j} /\left(P_{j}-1\right)>4$, then $T \geqq N-1$. It was found that $N=141$. From Lemma 2 we see that if $p^{a} \| m n$ and $p=5 k-1$ then $2 \mid a$. We conclude that $m n \geqq 5 \cdot 19^{2} \cdot 29^{2} \cdot F(7,1093)>4 \cdot 10^{354}$ where $F(7,1093)$ denotes the product of the 137 primes between 7 and 1093, inclusive, which are not congruent to -1 modulo 5 .

5. The case $(m n, 15)=3$. In this case $p_{1}=3$ and $p_{2} \geqq 7$. It follows that

$$
4<\prod_{i=1}^{T} p_{i} /\left(p_{i}-1\right) \leqq 1.5 \prod_{j=3}^{T+1} P_{j} /\left(P_{j}-1\right)
$$

so that if $N$ is the smallest integer such that $\prod_{j=3}^{Y} P_{j} /\left(P_{j}-1\right)>8 / 3$, then $T \geqq$ $N-1$. It was found that $N=54$. From Lemma 2 we see that if $p^{a} \| m n$ and $p=$ $3 k-1$ then $2 \mid a$. We conclude that $m n \geqq 3 \cdot 11^{2} \cdot 17^{2} \cdot 23^{2} \cdot G(7,571)>3 \cdot 10^{118}$. Here $G(7,571)$ is the product of the 49 primes between 7 and 571 , inclusive, which are congruent to 1 modulo 3 .

6. The case $(m n, 15)=15$. This case is far more troublesome and requires the examination of a multiplicity of subcases before the lower bound given in (d) of our theorem can be established. We shall present the results of our investigation in tabular form after some preliminary remarks.

We assume that $3^{a}\left\|m n, a \geqq 1 ; 5^{b}\right\| m n, b \geqq 1 ; 7^{c}\left\|m n, c \geqq 0 ; 11^{d}\right\| m n, d \geqq 0$; $13^{e}\left\|m n, e \geqq 0 ; 17^{f}\right\| m n, f \geqq 0 ; 19^{g}\left\|m n, g \geqq 0 ; 23^{h}\right\| m n, h \geqq 0 ; 29^{r} \| m n, r \geqq 0 ;$ $31^{s} \| m n, s \geqq 0$. Since $\sigma\left(3^{3}\right)=40$ and $5 \mid m n$ we see from Lemma 1 that $a \neq 3$. From Lemma 2 , since $15 \mid m n, b \equiv d \equiv f \equiv g \equiv h \equiv r \equiv 0(\bmod 2)$. Since $\sigma\left(7^{2}\right)=57$ and $\sigma\left(7^{3}\right)=400$, from Lemma 1 we have $c=0, c=1$, or $c \geqq 4$. Since $\sigma\left(19^{2}\right)=$ $3 \cdot 127, g=0$ or $g \geqq 4$. 


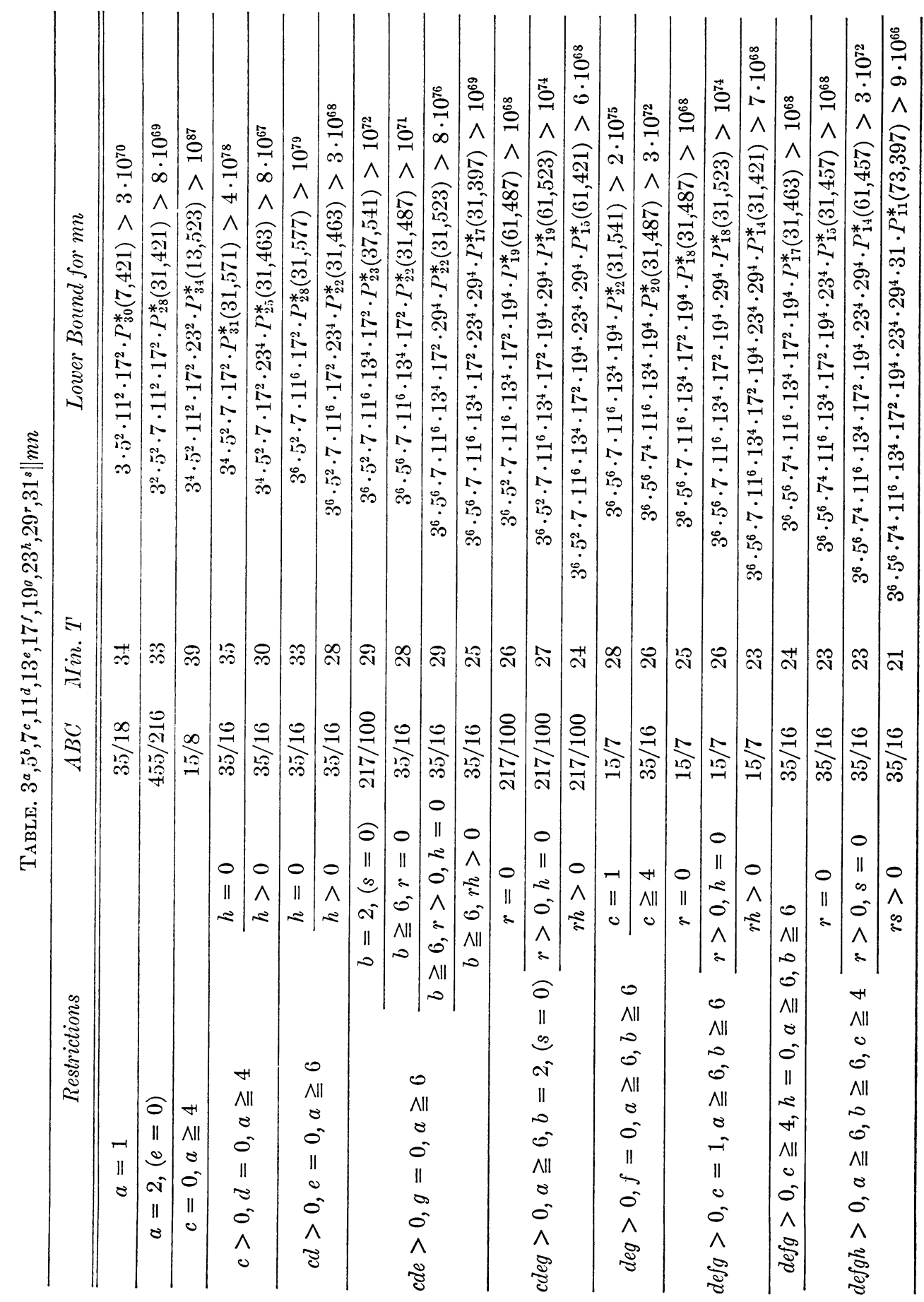


Now assume temporarily that $7 \mid m n$. Since $\sigma\left(3^{5}\right)=4 \cdot 7 \cdot 13, a \neq 5$. Since $\sigma\left(11^{2}\right)=$ $7 \cdot 19$ and $\sigma\left(11^{4}\right)=16105, d=0$ or $d \geqq 6$. Since $\sigma(13)=14, \sigma\left(13^{2}\right)=3 \cdot 61, \sigma\left(13^{3}\right)=$ $2380, e=0$ or $e \geqq 4$. Since $\sigma\left(23^{2}\right)=7 \cdot 79, h=0$ or $h \geqq 4$.

If $11 \mid m n$ then $a \neq 4$, since $\sigma\left(3^{4}\right)=121$; and $b \neq 4$, since $\sigma\left(5^{4}\right)=11 \cdot 71$. If $13 \mid m n$ then $r=0$ or $r \geqq 4$, since $\sigma\left(29^{2}\right)=13 \cdot 67$.

From Lemma 3 it follows that

$$
4<A B C \prod_{i=H}^{T} p_{i} /\left(p_{i}-1\right) \leqq A B C \prod_{j=H}^{R} P_{j} /\left(P_{j}-1\right) .
$$

Here $H=3$ if $7 \nmid m n, H=4$ if $7 \mid m n$. The asterisk indicates the (possible) omission of certain factors due to restrictions on $m n$. For example, if $17 \nmid m n$ then $17 / 16$ is omitted. If $5^{2} \| m n$ then, since $\sigma\left(5^{2}\right)=31,31 \nmid m n$ and therefore $31 / 30$ will be omitted. The number of factors in $\prod^{*}$ is $T-H+1 . A=\sigma\left(3^{a}\right) / 3^{a}$ if the value of $a$ is specified; $A=3 / 2$ otherwise. $B=\sigma\left(5^{b}\right) / 5^{b}$ if $b$ is specified; $B=5 / 4$ otherwise. $C=\sigma\left(7^{c}\right) / 7^{c}$ if $c$ is specified; $C=7 / 6$ otherwise.

From (4) we see that lower bounds for $T$, and consequently $m n$, can be determined by finding the smallest integer $N$ such that $A B C \prod_{j=I I}^{N^{*}} P_{j} /\left(P_{j}-1\right)>4$. Our results appear in the accompanying table.

In each case $P_{k}^{*}(p, q)$ denotes the product of the $k$ primes between $p$ and $q$, inclusive, which are not congruent to -1 modulo any prime known to be a divisor of $m n$. For example, in the last case

$$
P_{11}^{*}(31,397)=73 \cdot 127 \cdot 157 \cdot 163 \cdot 193 \cdot 211 \cdot 277 \cdot 283 \cdot 313 \cdot 331 \cdot 397 .
$$

None of these primes is congruent to -1 modulo $p$ where $3 \leqq p \leqq 31$.

In this case, since $\sigma(313)=2 \cdot 157$, we can improve the lower bound to $3^{6} 5^{6} 7^{4} 11^{6} 13^{4} 17^{2} 19^{4} 23^{4} 29^{4} 31 \cdot 73 \cdot 127 \cdot 157 \cdot 163 \cdot 193 \cdot 211 \cdot 277 \cdot 283 \cdot 331 \cdot 397 \cdot 421>$ $10^{67}$.

Since the cases discussed are mutually exclusive and exhaustive we conclude that $T \geqq 21$ and $m n>10^{67}$ if $15 \mid m n$.

7. Lower bounds for $m$ and $n$. Without loss of generality we assume that $m<n$. If $n<4 m$ then from our theorem we have $4 m^{2}>m n>10^{67}$. It follows that $m>10^{33}$.

If $n>4 m$ then from (1) we have $\sigma(m) / m=1+n / m>5$. Arguing as in Section 3 we see that if $N$ is the smallest integer such that $\prod_{j=1}^{N} P_{j} /\left(P_{j}-1\right)>5$ then $m$ has at least $N$ different prime divisors and $m \geqq \prod_{j=1}^{N} P_{j}$. It was found that $N=j 4$ and $m \geqq 3 \cdot 5 \cdots 257>8 \cdot 10^{102}$.

We have proved the following

CoRollaRy. If $m$ and $n$ are relatively prime odd amicable numbers then $m>10^{33}$ and $n>10^{33}$.

This improves Kanold's result [6] that $n>m>10^{23}$. We also remark that the adjective "odd" can be omitted in the statement of the corollary. For in [5] it has been proved that if $m$ and $n$ are relatively prime amicable numbers of opposite parity then $m>10^{36}$ and $n>10^{36}$.

Acknowledgements. The author would like to thank the referee for several 
helpful suggestions. He would also like to express his appreciation to Mr. John Bieler of the Temple University Computing Center for his cheerful cooperation.

Department of Mathematics

Temple University

Philadelphia, Pennsylvania 19122

1. J. Alanen, O. Ore \& J. Stemple, "Systematic computations on amicable numbers," Math. Comp., v. 21, 1967, pp. 242-245. MR $36 \# 5058$.

2. P. Bratley \& J. McKaY, "More amicable numbers," Math. Comp., v. 22, 1968, pp. 677678. MR $37 \# 1299$.

3. E. B. Escotr, "Amicable numbers," Scripta Math., v. 12, 1946, pp. 61-72. MR 8, 135.

4. M. GARCIA, "New amicable pairs," Scripta Math., v. 23, 1957, pp. 161-171. MR 20 \#5158.

5. P. HAGIS, JR., "Relatively prime amicable numbers of opposite parity," Math. Mag. (To appear.)

6. H.-J. KANOLD, "Untere Schranken für teilerfremde befreudete Zahlen," Arch Math., v. 4, 1953 , pp. 399-401. MR 15, 400.

7. E. J. LEE, "Amicable numbers and the bilinear diophantine equation," Math. Comp., v. 22, 1968 , pp. 181-187. MR $37 \# 142$.

8. P. Poulet, "43 new couples of amicable numbers," Scripta Math., v. 14, 1948, p. 77. 